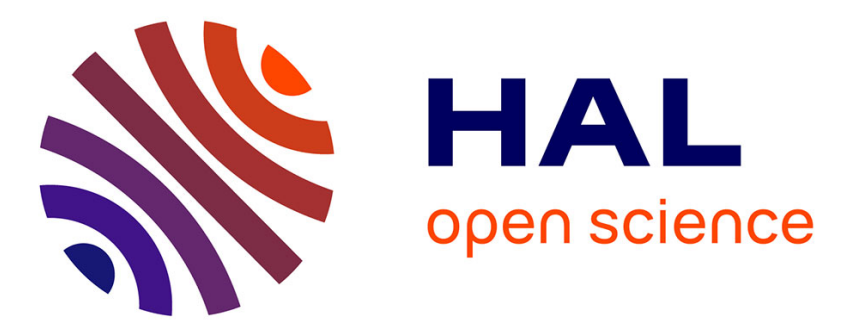

\title{
Numerical investigation on the electrical transmission ability of a shearing powder layer
}

Chaoqun Zeng, Mathieu Renouf, Yves Berthier, R. Hamdi

\section{To cite this version:}

Chaoqun Zeng, Mathieu Renouf, Yves Berthier, R. Hamdi. Numerical investigation on the electrical transmission ability of a shearing powder layer. Granular Matter, 2016, 10.1007/s10035-016-0619-z . hal-01347757

\section{HAL Id: hal-01347757 \\ https://hal.science/hal-01347757}

Submitted on 21 Jul 2016

HAL is a multi-disciplinary open access archive for the deposit and dissemination of scientific research documents, whether they are published or not. The documents may come from teaching and research institutions in France or abroad, or from public or private research centers.
L'archive ouverte pluridisciplinaire HAL, est destinée au dépôt et à la diffusion de documents scientifiques de niveau recherche, publiés ou non, émanant des établissements d'enseignement et de recherche français ou étrangers, des laboratoires publics ou privés.

\section{(c)(1)}

Distributed under a Creative Commons Attribution| 4.0 International License 


\title{
Numerical investigation on the electrical transmission ability of a shearing powder layer
}

\author{
C. ZENG - M. RENOUF - Y. BERTHIER - R. HAMDI
}

\begin{abstract}
Recent developments in powder technology gave birth to a new lubricant - powder lubricant. Compared to liquid lubricant, powder lubricant like graphite powder has several advantages, such as good electrical conductivity and good thermal resistance. Such advantages are especially appreciated in sliding electrical contacts. Thus, the study of the electrical transmission ability of a shearing powder layer under different dynamical constraints appears to have a great interest. Recent works allowed to model the coupling of mechanical and electrical effects in a discrete medium. This algorithm was extended to study the electrical properties of a shearing powder layer with Discrete Element Method. The mechanical and electrical behaviors of the sample were studied in different dynamical regimes, characterized by the inertial number $I$. The results exhibit an interesting relationship between the average contact resistance and the inertial number $I$. An exponential increase of the sample's electrical resistance as well as the induced electrical noise are observed closed to the dense flow limit. Such observations underline the fact that to ensure the electrical transmission ability of the powder layer, one must keep the particle size and shear rate small, and a sufficiently large pressure.
\end{abstract}

Chaoqun ZENG; Mathieu RENOUF

Laboratoire de Mécanique et Génie Civil (LMGC),

Université de Montpellier, CNRS, Montpellier, France

Tel.: +33-467149635

E-mail: mathieu.renouf@umontpellier.fr

Yves BERTHIER

Université de Lyon, LaMCoS,

INSA-Lyon, CNRS UMR5259, F-69621, France

Rachid HAMDI

Valéo système électrique, Isle d'Abeau, France
Keywords Electrical conductance - powder lubricant . Discrete Element Method

\section{Introduction}

Recent developments in powder technology have given birth to a new lubricant: powder lubricant. Compared to liquid lubricant, powder lubricants like graphite have several advantages such as static stress, good thermal resistance and electrical conductance [1]. Such advantages are especially appreciated in sliding electrical contact, since standard oil-based lubricant has poor electrical conductivity, and can not resist the Joule-heat generated by electrical current, which can rise to above $500^{\circ} \mathrm{C}$. In static case, where no shear motion occurred, many theories and models can be found in the literature, especially the pioneering works of Ragnar Holm [2], who had contributed in both theoretical and experimental sides. However, most works are limited to static cases, where no shearing occurred. Although the needs are emergent, very few investigations have been reported in a shearing sample. To fulfill these needs, the present paper proposes some results from numerical investigations on the mechanical and electrical coupling for the shearing powder, with 2D contact dynamic simulation. The mechanical and electrical coupling algorithm was developed by Renouf et al. [3] at the basis of works on multi-physical coupling in discrete element methods. The time-average resistance of a shearing sample in different regimes is studied, from quasistatic case to the limit of dense shearing [5] and compared to mechanical quantities such as the macroscopic friction or the volume fraction of the sample. 


\section{Simulation methodology}

\subsection{Mechanical framework}

Numerical simulations dedicated to the mechanical evolution of granular media can be based either on explicit $[4,5]$ or implicit $[6,7]$ method. The Non Smooth Contact Dynamics (NSCD) method used here is implicit and provides a non-smooth formulation of the bodies impenetrability condition, the collision rules, and the dry Coulomb friction law. The method is extensively described in Ref. [8] and briefly explained below.

Firstly, equations of motion are written for a collection of rigid bodies and discretized by a time integrator. The interaction problem is then solved at contact scale (local level) rather than at particle scale (global level) as commonly performed in explicit methods. In other words, equations are written in terms of relative velocities $\mathbf{u}_{\alpha}$ and local impulsions $\mathbf{r}_{\alpha}$ defined at each contact point indexed by $\alpha$. The impenetrability condition mentioned previously means that particle candidates for contact should not cross the boundaries of the antagonists bodies. Contacting bodies do not attract each other, i.e., that the reaction force is positive and vanishes when the contact vanishes. This can be summarized in the following so-called velocity Signorini condition:

$u_{n} \geq 0, \quad r_{n} \geq 0, \quad u_{n} r_{n}=0$

where the index $n$ denotes the normal component of the various quantities (index $\alpha$ is omitted). This philosophy is different from what is used in explicit methods [5], where normal forces are usually proportional to the penetration between two particles.

\subsection{Electrical computation}

For electrical computation, the algorithm developed by Renouf et al. [3] is used. The formulation of the electrical problem relies on an analogy between the contact network and an electrical resistance network. At any time $t$, it is supposed that the mechanical problem is solved and the interaction force between each contacting pair is known. For each contact, the electric current $I_{\alpha}$, induced by two neighboring particles, follows Ohm's law:

$I_{\alpha}=\frac{\Delta U_{\alpha}}{R_{\alpha}}$,

where $R_{\alpha}$ represents the contact pair resistance. $R_{\alpha}$ is determined by the classical electrical contact constriction model proposed by Holm [2]:

$R_{\alpha}=\frac{\rho}{2 a}$, where $\rho$ denotes the electrical resistivity of the particle material and $a$ the radius of the effective contact area. Such equation generally holds when $a<<r$ ( $r$ the radius of the particle), which is true for rigid discrete particles.

For small particles with high strain, which is typically the case in powder lubrication, the contact area is more likely to deform plastically than elastically. To calculate the apparent contact area $a$, Bowden et al.[9] propose the following formula:

$a=\sqrt{\frac{F}{\pi Y}}$

where $F$ is the contact force and $Y$ the yield strength. Such equation is valid for apparent contact area. The true contact area which allows electrical current passing is normally much smaller. Consequently the apparent contact area is multiplied by a factor $\gamma$ which represents the ratio between the effective contact area and apparent contact area. Such a parameter is identified through comparison with experimental data.

Once the local contacting model is chosen, the whole voltage network can be solved using the first Kirchhoff law for each particle $i$ :

$\sum_{\beta \in \mathcal{L}_{i}} I_{\beta}=0, \quad \forall i \in\{1, \ldots, N\}$,

where $N$ is the number of particles and $\mathcal{L}_{i}$ the list of contacts connected to particle $i$. Combination of Eq. (2) and (5) leads to the following linear system:

$\mathbb{C} \mathbf{I}=\mathbf{U}$,

where $\mathbb{C}$ is the conductivity matrix while $\mathbf{I}$ and $\mathbf{U}$ denote respectively the global intensity and electrical potential vectors. Combined given boundary conditions, such a linear system can be easily solved with a GaussSeidel algorithm [3].

To complete the model, simulations are compared to the experiments performed by Noda et al [10] to determine the value of $\gamma$ and to reproduce the linear relationship between the box height and the sample resistance. (cf. Fig. 1).

A value of $2.5 \times 10^{-2}$ is taken for $\gamma$ to obtain a good correlation between experimental and numerical result. It is interesting to point out that, since the periodic conditions in $x$ direction are used, the frictional effects between walls and the particle layer disappeared and the pressure is uniform on the lower boundary. For these reasons, the dependance of the electrical resistance on the height is still linear even for layer thickness larger than $8 \mathrm{~mm}$, contrary to the experimental data [10] for which the non-uniformity of the pressure is related to friction between particle layer and walls (Janssen effect). 


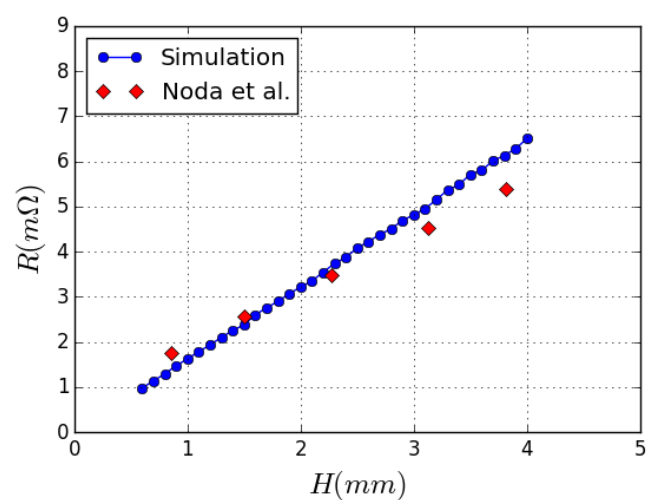

Fig. 1 Sample resistance $(R)$ vs box height $(H)$. Results were compared to the experimental data by Noda et al. [10]

\section{Shearing simulation}

\subsection{Numerical model}

In order to study the reliability of a powder lubricant used in sliding electrical contact, numerical investigation on the the electrical conductivity of a sheared powder sample are performed. The numerical material used in this section is identical to the one used in the previous section. The simulated system is two-dimensional (cf.Fig. 2).

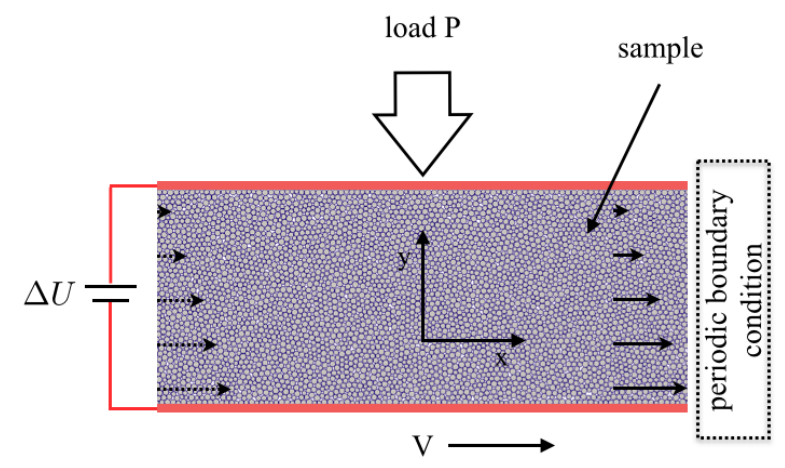

Fig. 2 Numerical sample for powder shearing with periodic boundary condition in the X-direction.

The granular medium is a dense assembly of 8641 rigid disks of average diameter $d$ equal to $200 \mu \mathrm{m}$ and average mass $m$. A small size polydispersity of $20 \%$ is considered to prevent crystallization [11]. The granular medium is submitted to a plane shear, without gravity, to obtain an uniform stress distribution. The material is sheared between two parallel rough walls of length $L$, distant from $H$. Periodic boundary conditions are applied along the X-direction. The wall's roughness is made with a set of grains sharing the same size polydispersity and mechanical properties than the flowing grains.
One of the walls is fixed, while the other moves at the given velocity $V$ which varies from $0.1 \mathrm{~m} / \mathrm{s}$ to $10 \mathrm{~m} / \mathrm{s}$ in order to give a wide range of velocity. The applied pressure $P$ varied from $250 \mathrm{~Pa}$ to $512 \mathrm{kPa}$. Both parameters $P$ and $V$ are kept constant in each simulation. Other important parameters can be found in Table 1.

Table 1 Simulation parameters (nominal values)

\begin{tabular}{ll}
\hline Average particle size $(r)$ & $100 \mu \mathrm{m}$ \\
Polydispersity $(\Delta r)$ & $20 \%$ \\
\hline Sample height $(H)$ & $8 \mathrm{~mm}$ \\
Sample width $(L)$ & $2 \mathrm{~cm}$ \\
\hline Load pressure $(P)$ & $0.25,512 \mathrm{kPa}$ \\
Velocity $(V)$ & $0.1,1,10 \mathrm{~m} / \mathrm{s}$ \\
Time step $(d t)$ & $1.0 \times 10^{-7} \mathrm{~s}$ \\
\hline
\end{tabular}

Moreover frictionless systems are considered leading to a zero value of local friction coefficient. Thus there is no rolling and the contact force has only a normal component. Account for friction will affect the normal component of the contact force and thus will change the effective contact area (Cf. Eq. (3)) which depends on the contact force. Nevertheless as the friction changes also the global volume fraction (compacity) of the medium as well as the whole contact network, considering a zero friction avoids such a competition and offers some perspectives to the present work.

\subsection{Inertial number $I$}

The inertial number, denoted $I$, measures the ratio of inertial force of grains to the imposed force: a small value corresponds to the quasi-static state, while a high value corresponds to the inertial (or dense flow) state or even the "dynamic" state [12]:

$I=\frac{\dot{\gamma} d}{\sqrt{P / \rho}}$,

where $\dot{\gamma}(=\mathrm{V} / \mathrm{H})$ is the shear rate, $d$ the average particle diameter and $\rho$ the density. Three regimes can be distinguished according to the value of $I$ : quasi static flow $\left(I<10^{-3}\right)$, dense flow $\left(10^{-3}<I<10^{-1}\right)$ and collisional flow $\left(I>10^{-1}\right)$.

\subsection{Mechanical aspect}

Figure 3 presents the visualization of the velocity field within the sheared sample.

Simulations are running until a steady state is reached. Such a steady state is generally associated to a linear 


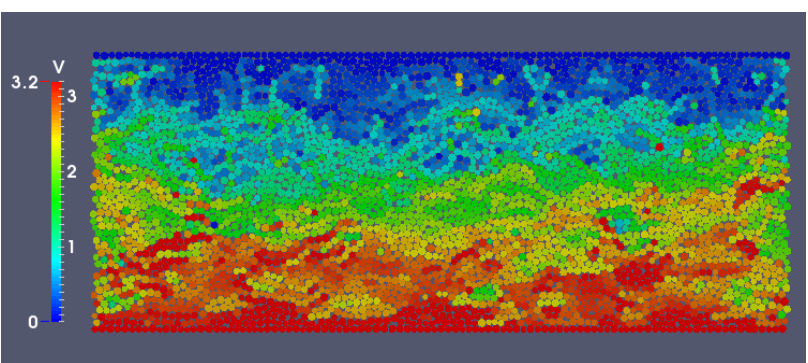

Fig. 3 Visualization velocity field within the sheared sample for a value of $I$ equal to 0.05 .

velocity profile through the thickness of the sample (Cf. Fig. 4a) (a)

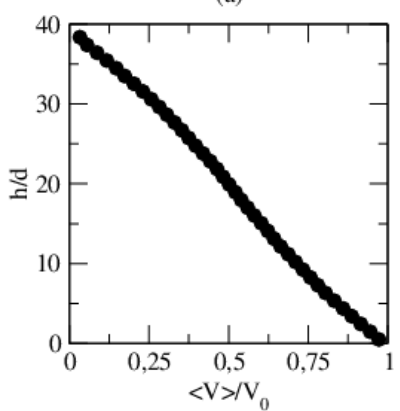

(b)

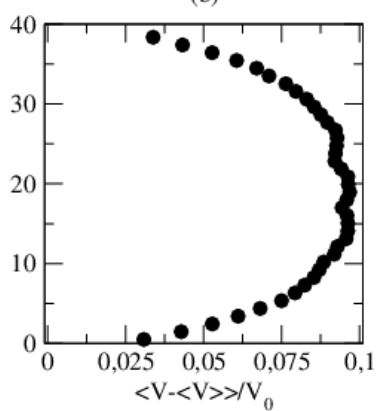

Fig. 4 (a) Velocity profile through the thickness of the sample and (b) velocity variation profile through the thickness of the sample

Indeed even if the profile is linear the system presents a shear band located in the center of the shear cell. As friction less contact are considered, it is not possible to observe the shear band using the angular velocity as in [14], but such a phenomena could be emphasize by plotting the fluctuation of the velocity around the mean velocity value (Cf. Fig. 4b).

In a quasi static flow, as its name indicates, the sheared granular layer is close to a static state. Its average packing fraction is then close to the RCP (Random Close Packing) limit. It remains true in dense flow regime, where the shearing rate would create more porosity then decrease the packing fraction, in order to better accommodate the dynamic strains. In the analytical model proposed by Dahmen et al. [15], authors have shown that voids in granular materials dissipate a fraction of the released stress.

Cruz et al.[13] show that the average packing fraction decreased proportionally to the inertial number $I$. So, the first step is to reproduce the same behaviors with varying stress $P$ and $V$, and the average particle size $r$. The instantaneous packing fraction is recorded by the upper plan position $y(t)$. By definition, the packing fraction $\Phi(t)$ is inversely proportional to $y(t)$. So the
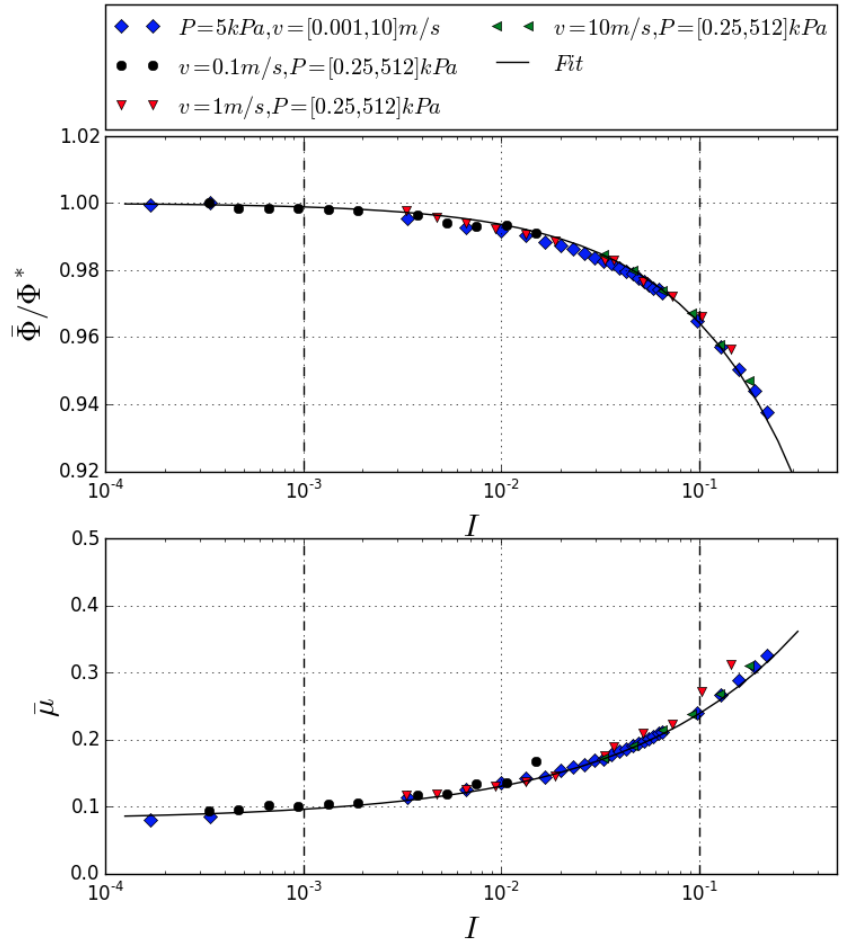

Fig. 5 Evolution of the time-average packing fraction $\bar{\Phi}$ and the effective friction coefficient $\bar{\mu}$ with increasing inertial number $I$. For $\Phi$ values were normalized to the static case where $I=0$. Varying dynamic stress have been applied with different values of velocity $V$ and applied pressure $P$. Data were compared to the linear equation proposed by Cruz et al. [13].

following relation can be drawn out:

$\frac{\Phi(t)}{\Phi^{*}}=\frac{y^{*}}{y(t)}$,

where $\Phi^{*}$ is the packing fraction for $V=0$ (or $I=0$ ), close to the RCP limit. The simulation results are summarized in Fig. 5. As expected, for values of $I$ smaller than $10^{-3}$, the relative packing fraction is close to 1 . For values of $I$ lying between $10^{-3}$ and $10^{-1}$, the increase is amplified. All data can be fitted by the linear equation proposed by Cruz et al. [13]:

$\Phi=\Phi^{*}(1-k I)$,

with $k=0.32$, close to the value $(0.3)$ proposed by the same author.

For the effective friction coefficient of the sample, another linear equation is proposed by Cruz et al. [13]. A similar trend is found here:

$\mu=\mu^{*}+b I$,

with $\mu^{*}=0.08$ and $b=1.2$. The slight difference of the static limit is related to the local friction condition used in [13]. However, it is interesting to note that the value of $b$ is unchanged and independent of the local friction. 


\subsection{Mean electrical resistance}

Concerning the electrical aspect of the simulations, it is possible to observe the evolution of the electrical potential within the sample and the regions with low or high resistivity (cf. Fig. 6). As no gravity is set on the simulation, blue dots on Figure 6 correspond to floating particles which have any contact.

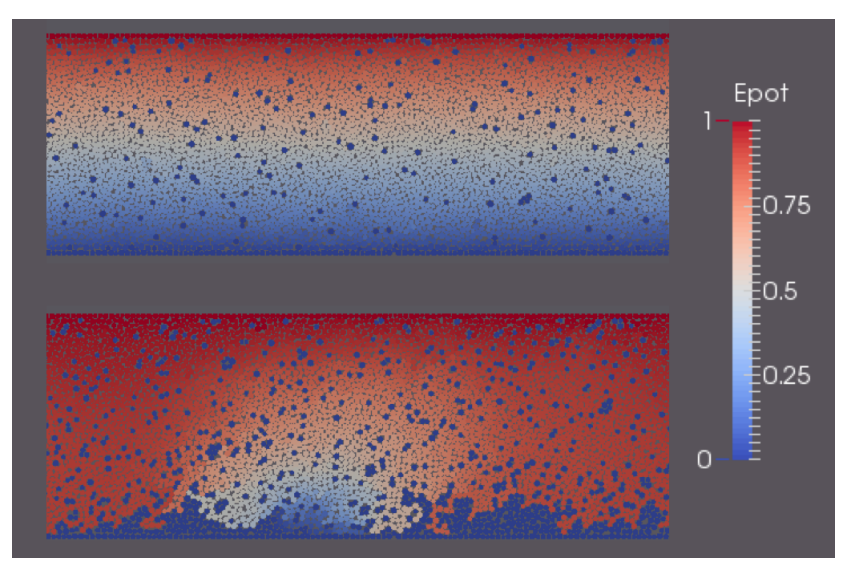

Fig. 6 Electrical potential map for $I=10^{-3}$ (up) and $I=$ 0.1 (down): The number of paths available to transmit the electrical current decreases for large values of $I$.

From a quantitative point of view, a general increase of the time-average electrical resistance of the sample $\bar{R}$ is observed with the increase of $I$ (cf. Fig. 7). Unlike the mechanical factors like $\Phi$ or $\mu$, the values of electrical resistance is plotted with logarithmic scale.

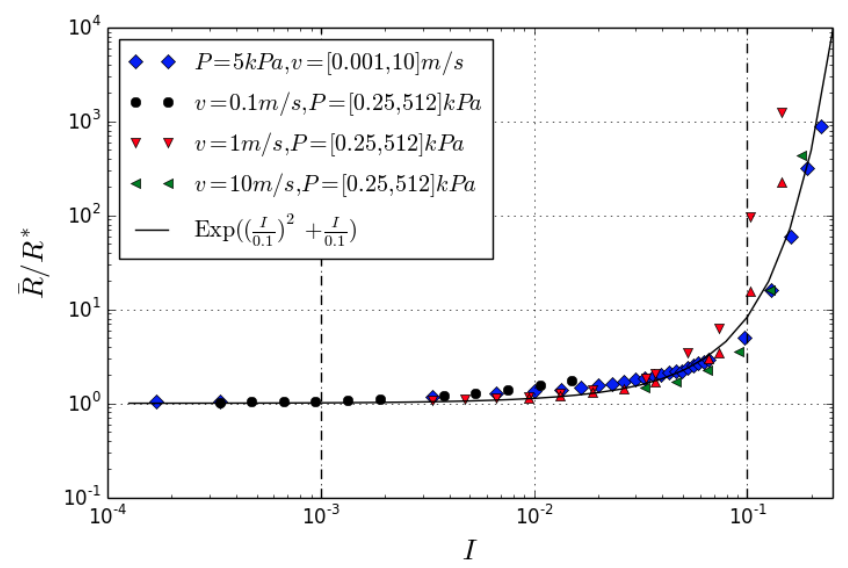

Fig. 7 Evolution of the time-average sample resistance $\bar{R}$ with increasing inertial number $I$. Values are normalized to the static case where $I=0$. Varying dynamic stress have been applied with different values of velocity $V$ and applied pressure $P$.

When $I$ get close to $10^{-1}$, at the edge of dense flow limit, a sharp increase of the resistance is observed. For
$I$ greater than $10^{-1}$, since very few or no percolation chains exist in the sample [16], the effective resistance becomes very large or even close to infinite. An exponential relationship can be found between $\bar{R} / R^{*}$ and $I$ :

$\frac{\bar{R}}{R^{*}}=\operatorname{Exp}\left(\frac{I}{I_{0}}\right)$

with $I_{0}=0.05$. Such equation is surprisingly simple, and its physical meaning is very interesting. Two lectures are possible. The first one is that, if the local kinematic is known (pressure and velocity), is it possible to determine the value of the average sample resistance. The second one is that, if it is possible to measure the average sample resistance, is it possible to determine the value of $I$ and to obtain some information concerning contact conditions. Nevertheless, one could observe that the fit for high values of $I$ is not as good as for small values (i.e. $I<410^{-2}$ ).

\subsection{Electrical noise}

In the analysis of an electrical signal, it's important no only to consider the average effective resistance, but also the noise amplitude which can represent a signature of the process. In some circumstances, the variation of a signal could be more important than the average effective resistance itself [17].

For this purpose, the temporal electrical signal is recorded during simulations (cf. Fig. 8).

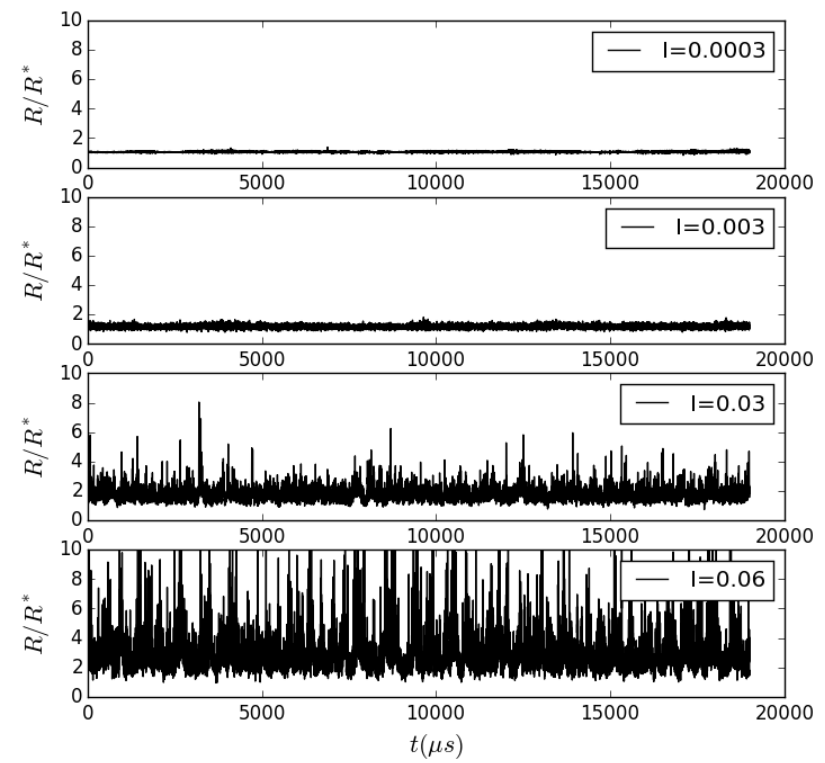

Fig. 8 Temporal sample resistance for different values of $I$.

As for the effective resistance, the shape of the temporal signal strongly depends on the inertial number 
$I$. In the quasi-static regime, the signal is smooth and exhibits small variations around its average value. In the dense flow regime, the signal becomes volatile and some pulses occur. To quantify the induced noise level with different values of $I$, the histogram of the signal is analyzed (cf. Fig. 9).

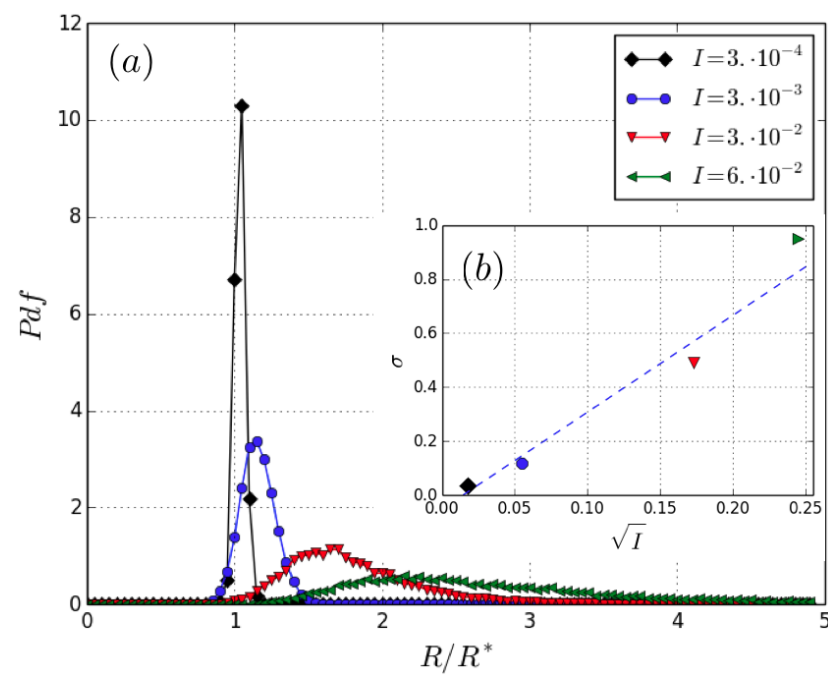

Fig. 9 (a) Histograms of temporal signal with some typical value of $I$ and (b) linear relationship between the standard deviation $\sigma$ and the square root of the inertial number $\sqrt{I}$.

One observe that the induced noise for small $I$ follows a normal-distribution. Such distribution suggests that the generated fluctuations are a white noise. It is interesting to point out that not only the average value of relative resistance varies with the inertial number $I$, but also the standard deviation $\sigma$. This last one seems to variate as a linear function of $\sqrt{I}$ : the higher is the inertial number the higher is the standard deviation.

As for the average value of relative resistance, the standard deviation $\sigma$ is plotted as a function of $I$ (cf. insert of Fig. 10). If the global trend is the same, it is difficult to fit all data. According to previous observation (cf. Fig. 9), such an observation seems logical, and if $I$ is kept as control parameter, it is the evolution of the product $\sigma \sqrt{V}$ which should be plotted as a function of $I$.

From Figure 10, it is possible to draw the following relation:

$\sigma \sqrt{V}=c\left(\operatorname{Exp}\left(\frac{I}{I_{c}}\right)-1\right)$

where $c$ and $I_{c}$ are respectively equal to 0.25 and 0.025 . Such results suggest that, not only the internal structure contribute to the noise level, but also the change rate $V$.

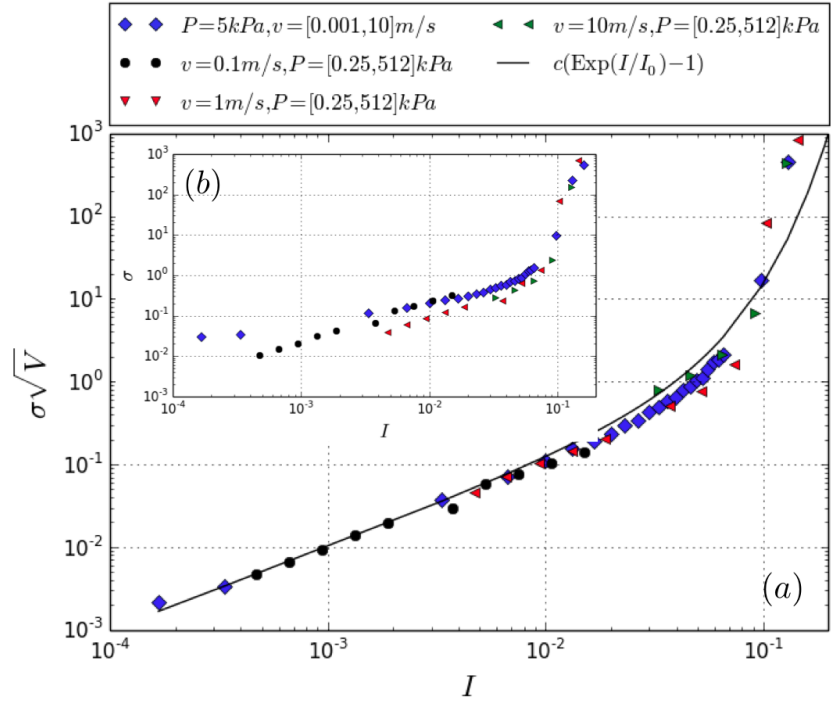

Fig. 10 Noise level (or standard deviation) $\sigma$ increases with inertial number $I$. Compared to raw data (b), a correction term $\sqrt{V}$ was found to be better fitted the simulation results (a).

\section{Conclusion}

The study of the electrical conductivity of a sheared powder sample is performed. Simulation results unveil the correlations between the mechanical and electrical phenomena in a sample under dynamical solicitations. Various aspects are analyzed in a dense shearing flow, including the packing fraction, the friction coefficient and the time-average electrical resistance with different boundary conditions.

Many papers from the literature suggested that the contact network depends strongly on the inertial number $I$ of the sheared sample, such as contact anisotropy [16], and the average coordination number [16]. As the electrical network is strongly related to the contact one, it is logical to obtain the dependency of the sample resistance on the inertial number $I$.

For a powder lubricated electrical contact, a low voltage drop is expected to limit electrical loss. With the present results, it can be seen that the inertial number $I$ in the system should be kept small all process long. It implies that for the same thickness, small powder particle, high pressure, and low shear rate are the key factors for a successful current transmission. So, contrary to liquid lubrication, where high velocity and low pressure are appreciated, high velocity is a threat to the electrical transmission for a powder lubricated electrical contact. Such results can guide engineers for their conception.

From another practical standpoint, since electrical signals are very reliable and sensitive, as we have shown, 
electrical signals could be used to detect events happening in a granular packing, just as many authors have already suggested [18]. Consequently, the proposed algorithm could give complementary informations.

\section{Disclosures}

This work was supported by the CIFRE grant 2013/0355. All figures in this paper have never been presented.

Acknowledgements Discrete element simulation have been carry out through open source software platform LMGC90 (https://git-xen.lmgc.univ-montp2.fr/lmgc90/lmgc90_user).

\section{References}

1. E.Y.a. Wornyoh, V.K. Jasti, C. Fred Higgs, ASME J. Tribol. 129(2), 438 (2007).

2. R. Holm, Electrical contact (1981)

3. M. Renouf, N. Fillot, Int. J. Numer. Meth. Engng 74(2), 238 (2008). DOI 10.1002/nme.2157.

4. P.A. Cundall, in Proceedings of the symposium of the international society of rock mechanics, vol. 1 (1971), vol. 1, pp. $132-150$

5. P.G. Rognon, J.N. Roux, M. Naaïm, F. Chevoir, J. Fluid Mech. 596, 21 (2008)

6. J.J. Moreau, in Nonsmooth Mechanics and Applications, ed. by J.J. Moreau, P. Panagiotopoulos (1988), pp. 1-82.

7. M. Renouf, H.P. Cao, V.H. Nhu, Tribol. Int. 44(4), 417 (2011).

8. M. Jean, Comput. Methods Appl. Mech. Engrg. 177(34), 235 (1999).

9. F.P. Bowden, D. Tabor, The Friction and Lubrication of Solids, 2nd edn. (Oxford University Press, Clarendon Press, 1986)

10. T. Noda, H. Kato, T. Takasu, A. Okura, M. Inagaki, Bull. Chem. Soc. Japan 39, 829 (1966)

11. S. Luding, Powders and Grains pp. 141-148 (2001)

12. F. Radjai, M. Jean, J.J. Moreau, S. Roux, Phys. Rev. Lett. 77(2), 274 (1996).

13. F. Cruz, S. Emam, M. Prochnow, J.N. Roux, F. Chevoir, Phys. Rev. E 72, 021309 (2005).

14. H.-J. Tillemans, H.J. Herrmann, Phys. A 217, 261 (1995).

15. K.A. Dahmen, Y. Ben-Zion, J.T. Uhl, Nature Physics 7(7), 554 (2011).

16. E. Azéma, F. Radjaï, Phys. Rev. Lett. 112(7), 078001 (2014).

17. M. Braunovic, V.V. Konchits, N.K. Myshkin, Electrical Contacts: Fundamentales, Applications and Technology (CRC Press, 2006)

18. L.J. Bredel, L.B. Johnson, D. Kuhlmann-Wilsdorf, Wear 120, 161 (1987) 